\title{
The impact of intermittent energy restriction and mastication on hippocampal cognitive ageing and neural stem cell fate: the change study - chewing, adult neurogenesis and energy restriction
}

This abstract was awarded the student prize.

Adult hippocampal neurogenesis (AHN), the postnatal generation of neurons in the dentate gyrus of the hippocampus, contributes to pattern separation, the differential encoding of small changes derived from similar inputs, and recognition memory, the recognition of previously encountered stimuli ${ }^{(1)}$.

Intermittent energy restriction (IER) and mastication have been shown to increase AHN and improve hippocampus-dependent cognition in rodents ${ }^{(2)}$. Previously we reported that IER and mastication interventions improved hippocampus-dependent memory in middle-aged adults ${ }^{(3,4)}$. We hypothesised that a 12-week IER and mastication intervention will improve hippocampus-dependent memory in overweight, ageing human adults. Furthermore, the impact on the systemic milieu will lead to changes in the neurogenic process in vitro.

A 12-week parallel 4-arm randomised controlled trial (ethical approval from KCL research ethics committee HR-17/18-5407) was conducted in 89 healthy, overweight human adults aged $\geq 60$ years to investigate the impact of IER ( $2.51 \mathrm{MJ} / \mathrm{day}$ for 2 days per week via nutritionally complete meal replacement products) and mastication (1 piece sugar-free chewing gum 3 times a day) compared with control (no intervention), independently and synergistically, on hippocampus-dependent cognition and the anti-ageing protein Klotho which has been implicated in IER and AHN. Random effects linear mixed modelling was used. Dietary intake, body composition, mood, and sleep quality were also explored. The effect of the interventions on the systemic milieu and consequential impact on the neurogenic process was also investigated using serum-treated hippocampal progenitor cells (HPCs). Cellular readouts of proliferation, stem cell integrity, differentiation and apoptosis were compared between interventions. Baseline correlations between dietary intake and hippocampus-dependent cognition with the neurogenic process were also explored.

There was no overall effect of IER and mastication on hippocampus-dependent cognition in comparison to control. However, the intervention groups had modest within-group improvements in pattern separation in the absence of a corresponding effect in vitro on the neurogenic process. IER led to a reduction in weight compared to control $(-2.85 \mathrm{~kg}, 95 \% \mathrm{CI}[-4.12,-1.05], P<0.001)$.

Moreover, IER in combination with mastication led to reductions in saturated fat intake compared to control $(-8.21 \mathrm{~g} / \mathrm{day}, 95 \% \mathrm{CI}$ [15.53, -0.89$], P=0.019)$ and free sugar intake compared to mastication only $(-12.55 \mathrm{~g} / \mathrm{day}, 95 \% \mathrm{CI}[-24.17,-0.92], P=0.027)$ possibly explaining the observed cognitive improvements. Baseline serum concentration of Klotho and fat intake was negatively associated with HPC proliferation. Finally, greater baseline consumption of folate, vitamin E and selenium were associated with increased survival of proliferating HPCs.

To conclude, IER and mastication may benefit the ageing-sensitive hippocampus dependent cognition in ageing humans. Moreover, preliminary results suggest that specific micronutrients and the anti- ageing protein Klotho are related to the human neurogenic process. Larger human trials and future in vitro experiments are required to further understand and validate these findings.

\section{References}

1. Clelland CD, Choi M, Romberg C et al. (2009) Science 325, 210-3.

2. Murphy T, Dias GP \& Thuret S (2014) Neural Plast 2014, 1-32.

3. Kim C, Miquel S \& Thuret S (2019) Nutr Heal Aging 5, 33-42.

4. Kim C, Pinto AM, Bordoli C et al. (2020) Nutrients 12, 638. 